\title{
Corela
}

Cognition, représentation, langage

HS-11 | 2012

RJC Cotexte, contexte, situation

\section{La couverture médiatique de la « crise des banlieues » : métaphores, représentations et l'apport indispensable du cotexte}

\section{Stéfanie Peeters}

\section{(2) OpenEdition}

Journals

Édition électronique

URL : http://journals.openedition.org/corela/2079

DOI : $10.4000 /$ corela.2079

ISSN : 1638-573X

Éditeur

Cercle linguistique du Centre et de l'Ouest - CerLICO

Référence électronique

Stéfanie Peeters, «La couverture médiatique de la « crise des banlieues » : métaphores,

représentations et l'apport indispensable du cotexte », Corela [En ligne], HS-11 | 2012, mis en ligne le

02 avril 2012, consulté le 10 décembre 2020. URL : http://journals.openedition.org/corela/2079 ; DOI : https://doi.org/10.4000/corela.2079

Ce document a été généré automatiquement le 10 décembre 2020.

\section{cc) (i) (2) (2)}

Corela - cognition, représentation, langage est mis à disposition selon les termes de la licence Creative Commons Attribution - Pas d'Utilisation Commerciale - Partage dans les Mêmes Conditions 4.0 International. 


\title{
La couverture médiatique de la « crise des banlieues ": métaphores, représentations et l'apport indispensable du cotexte
}

\author{
Stéfanie Peeters
}

L'auteure tient à remercier le Fonds de la Recherche Scientifique - Flandres (FWO) pour avoir financé son projet de doctorat, dans lequel cadre le présent article.

\section{Introduction}

1 Comme le pose Fowler (1991: 4), « les nouvelles [sont] la représentation du monde en langue » (notre traduction). Sur la base de cette idée, nous avons entamé l'analyse des nouvelles parues dans la presse quotidienne nationale lors de « la crise » des banlieues en 2005, dans le but de révéler les représentations qui circulent dans le débat public français sur les banlieues, leurs habitants, les jeunes de banlieues et leurs difficultés (cf. e.a. Boyer et Lochard 1998 ; Peralva et Macé 2002; Djordjević 2007 ; Garcin-Marrou 2007 ; Sedel 2009). Plus particulièrement, nous avons compilé un corpus consistant en tous les articles traitant des « violences urbaines " ${ }^{1}$ dans trois journaux nationaux dits « de qualité » (Le Figaro, Libération et Le Monde) et un journal dit « d'opinion » (L'Humanité) (cf. Charon 2005).

2 Lors de l'examen de ce corpus de presse, le focus a particulièrement été mis sur l'usage des métaphores, étant donné que la métaphore est une "stratégie discursive » spécifique (Wodak et De Cillia 2006 : 717) dont un locuteur peut se servir pour évoquer et, du coup, représenter certains événements et acteurs. En effet, un journaliste qui parle, comme en (1), des émeutes dans les banlieues en termes d'une "contagion » (" émeutes » étant le soi-disant topic de la métaphore, " contagion » étant le dit véhicule) semble proposer aux lecteurs une image bien particulière des événements ainsi que des émeutiers impliqués :

(1) [TITRE] Emeutes : Paris se protège de la contagion. (Le Figaro, 12/11/2005_1) 
Autrement dit, si « les objets du discours doivent être éclairés, ce qui revient à mettre en évidence quelques-unes de leurs facettes et à en occulter d'autres » (Grize 2005 cité dans Moirand 2006: 16), l'usage de métaphores peut y contribuer de façon considérable. Plus particulièrement, depuis que Lakoff et Johnson (1980) ont formulé leur théorie de la métaphore conceptuelle, la métaphore est généralement conçue comme un «outil de représentation", un "outil pour construire une réalité", un "outil de cadrage " ( « framing device »; Pan et Kosicki 1993; Chilton 2004 ; Lakoff 2004 ; Van Gorp 2005). En effet, d'après ces deux chercheurs cognitivistes, la métaphore consiste à construire un domaine conceptuel (le domaine cible) en termes d'un autre (le domaine source). En conséquence, le choix de ce dernier aura une influence sur la façon dont le domaine cible sera représenté, "cadré », voire évalué. Etant donné cette "fonction de représentation » (cf. Koller 2003, 2004) des métaphores, une inventorisation des métaphores dans les articles traitant des «violences urbaines» de 2005, pourrait selon nous être particulièrement indicatrice et révélatrice des représentations courantes des banlieues dans la sphère publique française de l'époque.

Or, quoique la théorie de la métaphore conceptuelle de Lakoff et ses collègues soit souvent utilisée pour mettre en rapport métaphores linguistiques et représentations conceptuelles ${ }^{2}$, cet article plaidera, dans le droit fil d'une tendance récente dans les études des métaphores (e.a. Eubanks 2000 ; Cameron 2003, 2007 ; Koller 2003, 2004), en faveur d'une approche des métaphores qui est plus pragmatique et plus axée sur le cotexte. Nous démontrerons qu'une telle perspective cotextuelle est plus à même de rendre compte des associations évaluatives (vis-à-vis des banlieues et des émeutiers, à savoir victimisantes versus culpabilisantes; cf. infra) caractéristiques de mainte métaphore dans notre corpus.

Pour ce faire, nous commencerons par montrer à quel point la théorie de la métaphore en général est dominée par une vision dite «bifurquée » (Eubanks 2000: 13-29) sur les métaphores et que cette perspective binaire connaît son apogée lorsque les métaphores sont vues, dans le sillage de Lakoff et Johnson (1980), comme des " outils de cadrage». Dans un deuxième temps, nous tenterons d'illustrer à l'aide d'une discussion des spécificités de notre corpus (cf. partie 3), des métaphores qui y apparaissent (cf. partie 4) et des occurrences de la métaphore spécifique ghetto (cf. partie 5) qu'une telle vision bifurquée est loin de suffire à elle seule pour rendre compte des représentations que peuvent véhiculer les métaphores dans notre « corpus des banlieues ».

\section{L'approche courante, « bifurquée » des métaphores}

6 Depuis qu'Aristote a prêté attention au phénomène de la metaphora, divers théoriciens dans ce domaine ont considérablement élaboré, adapté et raffiné la théorie de la métaphore ${ }^{3}$, sans que l'on ait toutefois abandonné l'idée que la métaphore est une expression binaire ${ }^{4}$. En effet, selon le cadre théorique spécifique, la métaphore consiste en une teneur et un véhicule (tenor et vehicle; Richards 1936), un sujet primaire et secondaire (primary et secondary subject; Black 1979) ou un domaine cible et un domaine source (Lakoff et Johnson 1980, 1999) (les termes sont grosso modo interchangeables) (cf. Eubanks $2000: 16)$.

7 De la même façon, dans les études où le focus est mis sur les représentations que véhiculent les métaphores et où l'aspect de «cadrage » des métaphores entre en jeu, la 
perspective bifurquée des métaphores et l'interaction entre les deux composantes de la métaphore (le domaine source, d'une part, et le domaine cible, d'autre part) semblent également jouer un rôle-clé : les métaphores ne cadreraient les topics d'une certaine façon que parce que le domaine source de la métaphore (premier élément de la bifurcation) permet de projeter certaines inférences au domaine cible (seconde composante de la bifurcation). Les citations suivantes montrent à quel point il est courant d'attribuer la fonction "cadrante" des métaphores (c.-à-d. leur fonction de représentation) à leur domaine source et aux inférences que ce dernier facilite :

Metaphorical expressions are particularly involved in the function of representation, since their interpretation involves the projection of material from source to target domains, including particularly patterns of inferences (Chilton 2004: 51-52; nous soulignons)

The choice of one metaphor rather than another [c.-à-d. le fait de préférer un domaine source à un autre] has consequences for how a particular issue is 'framed' or structured, which aspects are foregrounded and which backgrounded, what inferences are facilitated, what evaluative and emotional associations are triggered, what courses of action appear to be possible and so on. (Semino 2008: 91; nous ajoutons)

8 En effet, dans les extraits susmentionnés, l'on suggère que le domaine source de la métaphore est à même de provoquer une représentation spécifique d'un topic, en ce qu'il permet la projection de certain matériel (inférences, associations évaluatives et émotionnelles) au domaine cible.

Cependant, à notre avis, la tendance à considérer les métaphores comme des expressions binaires risque de déboucher sur une attention trop exclusive au (voire une surestimation du) rôle du domaine source pour la représentation véhiculée, au détriment de l'influence que peut avoir le cotexte plus large de la métaphore en question.

En effet, en essayant d'appliquer cette perspective théorique binaire aux métaphores dites « at work » de notre corpus pour examiner les représentations que ces métaphores véhiculent, il nous est devenu clair que la nature des métaphores est souvent trop complexe pour rentrer dans une structure binaire: les métaphores interagissent constamment avec d'autres éléments du discours (le cotexte), interaction qui exerce inévitablement une influence sur la représentation véhiculée. Avant que nous puissions illustrer cette idée à l'aide d'extraits métaphoriques concrets, il nous faut nous attarder sur quelques spécificités de notre corpus.

\section{Le " corpus des banlieues » : opposition au niveau des représentations}

11 Comme notre analyse se focalise sur les nouvelles publiées lors d'une situation de crise dans les banlieues dans quatre journaux aux lignes éditoriales variées ${ }^{5}$, il nous semble inévitable que les journalistes des articles sous examen s'engagent dans la «lutte de définition de 'la banlieue'" , dans le « conflit de définitions » qui caractérise généralement le débat sur les banlieues (Peralva et Macé 2002: 11; Sedel 2009: 27). En effet, les journalistes de l'époque se trouvaient dans la position délicate de ne pas seulement renseigner les lecteurs sur les événements violents dans les banlieues, mais aussi de les expliquer, voire de leur trouver une causalité. Comme l'illustre la figure 1 (adaptée d'après Djordević 2007), ce concept de causalité peut être visualisé à l'aide d'un axe opposant un pôle de responsabilité à un pôle de réactivité, lesquels pôles peuvent être 
occupés soit par des acteurs institutionnels (hommes politiques, policiers,...), soit par des acteurs non institutionnels (habitants des banlieues, émeutiers,...).

Figure 1 : deux univers liés par l'axe de causalité

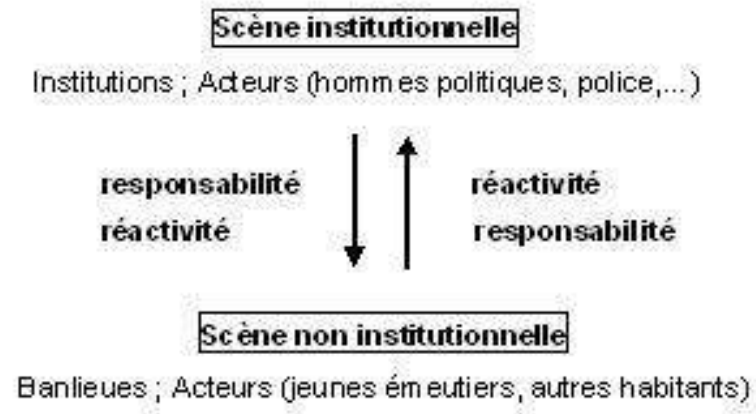

En simplifiant les choses, nous pourrions poser que certains énonciateurs dans notre corpus $^{6}$ tiennent les hommes politiques et leur politique de la ville boiteuse pour responsables (pôle de responsabilité) de la vie inhumaine dans les banlieues, à laquelle les émeutiers urbains ne font que réagir (pôle de réactivité), tandis que d'autres énonciateurs voient les acteurs urbains comme les responsables des événements, tout en attribuant le rôle de réacteurs aux hommes politiques.

Les différences entre les textes journalistiques au niveau du positionnement des divers acteurs impliqués sur l'axe de causalité résultent, d'après nous, en une opposition au niveau des représentations des banlieues et des émeutiers au sein du corpus. En effet, nous pouvons poser que notre corpus fait preuve d'une opposition entre, grosso modo, deux types de représentations : d'une part, nous repérons des textes qui véhiculent une représentation victimisante des jeunes/banlieues et, d'autre part, nous en trouvons qui amènent une représentation plutôt culpabilisante. Le premier type d'articles opte pour la compréhension, en défendant que les banlieues ne sont que des victimes (et occupent, du coup, le pôle de réactivité de l'axe de causalité), réagissant à une panoplie d'injustices (chômage, précarité, relégation, discriminations, etc.) qui leur sont faites d'en haut (pôle de responsabilité). Le second groupe, par contre, semble altériser («othering»; cf. Coupland 1996) les banlieues et les émeutiers, en mettant l'accent sur la responsabilité et le manque de respect des jeunes et en accordant le rôle de réacteurs (pôle de réactivité) aux hommes politiques et aux policiers ${ }^{7}$. Tandis que la presse de gauche (Libération et L'Humanité) contient pas mal de représentations victimisantes des jeunes, la perspective de la culpabilisation est très présente dans Le Figaro, le journal de droite dans notre corpus (cf. Garcin-Marrou 2007)

Toutefois, il nous faut également admettre que le corpus contient des textes qui font exception à cette tendance globale de victimisation/culpabilisation, et semblent opter pour une représentation plus neutre et nuancée. En d'autres termes, la bipolarité n'est pas catégorique ou binaire à cent pour-cent et permet des positions intermédiaires : la culpabilisation et la victimisation ne sont que rarement totales et se combinent plutôt dans des proportions à établir dans chaque cas particulier. Les articles du Monde sont à ce sujet des cas intéressants, comme l'on y retrouve les deux cadres représentatifs (victimisation et culpabilisation) : l'on y cadre les événements de façon assez nuancée et équilibrée, ayant l'œil pour des aspects sociaux qui permettent une certaine victimisation 
des jeunes, sans pour autant décharger les jeunes de leur responsabilité ou s'abstenir de considérer les violences comme inacceptables.

C'est dans ce corpus aux points de vue majoritairement polarisés que nous sommes allée à la recherche des métaphores qui nous renseignent sur le topic des banlieues et des émeutiers.

\section{Représentation victimisante versus culpabilisante : vers un duel des cadres métaphoriques?}

Comment les métaphores se comportent-elles maintenant dans cette opposition de représentations qui caractérise notre corpus? Autrement dit, à quel point les deux perspectives contrastantes correspondent-elles à une opposition au niveau du cadrage métaphorique? Notre analyse du corpus nous permet de formuler deux grandes tendances, à ce sujet :

D'une part, notre analyse a révélé des occurrences métaphoriques, à travers lesquelles une représentation bien particulière (soit victimisante, soit culpabilisante) est véhiculée, puisque le "cadre » (domaine source) évoqué impose, de façon intrinsèque, une structure bien spécifique et des connotations bien particulières qui mènent assez univoquement à la culpabilisation ou bien à la victimisation du topic en question. Aussi, dans ce groupe, est-ce le cadre évoqué qui joue un rôle prépondérant dans la représentation que véhicule la métaphore. Dans les journaux de gauche, par exemple, les journalistes ou les autres voix ayant la parole, optent souvent pour des domaines source qualifiables de "réactifs ", tels que PAIEMENT ou RÉPONSE, en parlant des émeutes. C'est notamment le cas pour les métaphores (indiquées en gras) en (2) et (3):

(2) Pour François Chérèque, secrétaire général de la CFDT et ancien éducateur spécialisé, on est "en train de payer ", avec la crise dans les banlieues, "vingt ou vingt-cinq ans » de " désinvestissement de l'Etat " (Libération, 08/11/2005_11; nous soulignons)

(3)[TITRE] Invité de la semaine: Mustapha Sandid. Étudiant, vivant à Bobigny (Seine-Saint-Denis). «Face à un État policier qui utilise la répression au lieu de prôner le dialogue, une partie des jeunes ont décidé de répondre par la violence. » [...] À force de subir tout cela et face à un État policier qui utilise la répression au lieu de prôner le dialogue, une partie des jeunes ont décidé de répondre par la violence, la seule véritable solution à leur sens : brûler des voitures et saccager des écoles, telle est leur arme face à l'État qui les méprise... Brûler pour s'exprimer, utiliser la violence pour exister dans cette société. (L'Humanité, 07/11/2005_13; nous soulignons)

18 En utilisant des métaphores qui ressortissent à ces domaines source spécifiques (PAIEMENT et RÉPONSE), l'on présente les violences comme ayant une cause externe, comme étant une réaction à quelque chose d'autre. En effet, on n'a "paiement » que parce qu'il existe une chose qui doit être payée (en l'occurrence, « vingt ou vingt-cinq ans» de "désinvestissement de l'État»). De la même façon, on n'a réponse que parce qu'on réagit à une phrase antérieure (« un État policier qui utilise la répression au lieu de prôner le dialogue »). En d'autres termes, c'est sous l'influence du domaine source des métaphores en question qu'une représentation victimisante des émeutiers est véhiculée.

19 D'autre part, nous avons rencontré des métaphores où c'est le cotexte, plutôt que le domaine source évoqué par le terme métaphorique en soi, qui détermine (ou guide) la fonction de représentation de la métaphore. En effet, notre corpus contient plusieurs 
métaphores qui ressortissent à des domaines source identiques (et sont souvent même formellement identiques), tels que le domaine de GUERRE, de FEU, de MALADIE. Toutefois, les expressions métaphoriques qui puisent dans ces domaines source véhiculent une représentation tantôt victimisante, tantôt culpabilisante, suivant le cadre argumentatif dominant l'article entier, comme nous allons le montrer par la suite. Plus particulièrement, il semble que le type de représentation véhiculée (victimisante versus culpabilisante) soit souvent déterminé par la façon dont le cotexte nous donne des indices sur le positionnement des acteurs du débat (hommes politiques, jeunes, police,...) sur l'axe de causalité (avec ses pôles de responsabilité/culpabilité et réactivité).

Dans la section suivante, nous analyserons quelques exemples spécifiques, dans le but d'illustrer ce second groupe de métaphores.

\section{Un exemple symptomatique : le cas des banlieues- ghettos}

21 Pour exemplifier le second type de métaphores, nous aimerions présenter le cas de ghetto, métaphore à l'aide de laquelle pas mal de passages du corpus décrivent les banlieues. Force est de constater que ce mot comporte par nature des connotations négatives, en raison de sa lourde charge historique.

Toutefois, pour ce qui est de la représentation des banlieues (ce qui est, rappelons-le, notre focus d'intérêt) que la métaphore ghetto véhicule, celle-ci n'est point univoque et dépend du cotexte de cette métaphore et du patron d'argumentation qui s'y développe. Premièrement, certains cotextes mettent l'accent sur la passivité des banlieusards dans la création des ghettos et sur l'existence de causes exogènes qui en expliquent l'existence. Bref, le pôle de responsabilité de l'axe de causalité, tout divers que l'élément exact qui l'occupe puisse être, se trouve cotextuellement rempli par des acteurs institutionnels, ce qui mitige, d'après nous, la représentation que véhicule la métaphore ghetto des banlieues mêmes. Ainsi, dans les exemples (4)-(7), sortis de L'Humanité et Libération, diverses voix attribuent la ghettoïsation à des facteurs externes :

(4) [Journaliste :] Ces événements signent-ils l'échec de trente ans de politique de la ville? [Manuel Boucher :] C'est une question complexe. Car la politique de la ville peut être présentée comme un simple pansement sur une jambe de bois, tout juste destinée à éviter les explosions les plus graves. En même temps, elle a permis des avancées intéressantes, en terme d'éducation avec les zones d'éducation prioritaires (ZEP), en terme de logement avec de nouvelles réflexions sur la façon de faire évoluer le bâti, en terme de recomposition du tissu socio-associatif. Mais, c'est vrai, la politique de la ville n'a pas réellement permis aux habitants de s'émanciper et de prendre en main la vie de leur quartier. Car ceux qui la mènent, cette politique, pour l'essentiel, ce sont des «chargés de projet » qui viennent de l'extérieur et font avant tout de la communication. Vue comme cela, la politique de la ville n'est plus qu'un moyen d'encadrer les classes populaires. Et de continuer à les concentrer dans des ghettos. (L'Humanité, 05/11/2005_8; nous soulignons et ajoutons)

(5) Peu à peu, le cortège se met en marche, direction le quartier du Chêne-Pointu. Des carcasses de voitures calcinées barrent la rue. Sur les murs des HLM, une phrase est taguée : " Bouna, on t'aime. " Dans les rangs, la colère est palpable. " Je comprends ces jeunes qui se sentent exclus, enfermés dans des ghettos. À Clichy, on fait tout pour qu'ils s'en sortent, mais les propos de Sarkozy attisent leur colère. Nous, on nettoie nos poubelles au Kärcher, pas nos cités ", souligne Claudine 
Thévenot. (L'Humanité, 03/11/2005_1; nous soulignons)

(6)[SOUS-TITRE] «Le gouvernement ignore la situation réelle» [...] Depuis vingt ans, on parle d'intégration, on demande à des gens de s'assimiler à la société, à la culture française, mais derrière il n'y a pas de partage, de travail de création d'une culture commune. C'est ainsi que petit à petit on a créé du communautarisme, de la ghettoïsation. (L'Humanité, 04/11/2005_3 ; nous soulignons)

(7)[TITRE] Mots de ghetto. Des jeunes des cités racontent à "Libération " les raisons de leur colère, le chômage, les cités délabrées, les contrôles de police. Et leur haine de Sarkozy. p. 2 à 4 (Libération, 05/11/2005_1; nous soulignons) verbe actif «concentrer» combiné avec le sujet «la politique de la ville» (mesure institutionnelle) et avec le COD «les» (renvoyant aux «classes populaires») signale qu'un facteur externe ( la politique de la ville ») transforme les banlieues en ghettos. En (5), la présence des participes passés "exclus » et "enfermés " auprès de "ghettos " indique également que ces derniers ne sont point des créations des jeunes mêmes, mais d'autrui (celui qui enferme, celui qui exclut). Dans l'extrait (6), le maire PCF de Sevran a la parole. Il dit, lui aussi, explicitement qu'« on a créé [...] de la ghettoïsation», le verbe « créer » impliquant également un acteur externe spécifique, à savoir le gouvernement. En (7), fragment à la Une de Libération, enfin, le journaliste énumère quelques éléments qui font que les banlieues soient des ghettos: «le chômage, les cités délabrées, les contrôles de police ", tous des éléments qui suggèrent une cause de ghettoïsation externe. En d'autres termes, si les banlieues sont bel et bien des ghettos dans (4)-(7), ce n'est pas de leur faute. En conséquence, la métaphore ghetto résulte ici en une représentation négative des hommes politiques au pouvoir, plutôt que des banlieues mêmes.

D'autres cotextes, par contre, soulignent justement à quel point les banlieusards sont eux-mêmes responsables de leurs ghettos (causes endogènes, telles que l'insécurité, la criminalité,...), ce qui débouche sur une représentation plus négative des banlieues. Ainsi, dans les fragments (8)-(9), soutenant, eux aussi, que les banlieues sont des ghettos, la responsabilité de cette ghettoïsation est attribuée à des forces internes aux banlieues. Du coup, ils présentent une perspective culpabilisante des jeunes. Si les extraits (4)-(7) provenaient de L'Humanité et de Libération, ces exemples concernent tous des fragments du Figaro :

(8) Aveuglés et tétanisés par les bobos salonnards et bien-pensants, nos gouvernements vont-ils se rendre à l'évidence ? Vont-ils enfin prendre la mesure que ces jeunes, Français malgré eux, issus d'une immigration arabo-africaine, entendent garder leurs spécificités culturelles et religieuses? Loin de vouloir se fondre et s'intégrer dans une France timorée qui confond tolérance et laxisme, ils se ressourcent continuellement dans leurs origines toutes proches grâce aux moyens de communication moderne et refusent de sortir de leur ghetto identitaire. Aujourd'hui, le cocktail entre la haine des Français et la culture du ghetto refermé sur lui-même explose et ses ravages continueront à se propager et à s'amplifier. (Le Figaro, 04/11/2005_13; nous soulignons)

(9) Membres du conseil syndical, ils veillent depuis toujours à leur cadre de vie quotidien. « Avec Jean-Jacques et les autres, on avait tout repris en main, commente Jean-Pierre Moreau, 58 ans. Comme ça commençait à devenir un peu " ghetto » ici, on avait effacé les graffitis, transformé un terrain vague en coin poubelles et même donné des consignes au concierge. » (Le Figaro, 08/11/2005_12 ; nous soulignons)

Ainsi, le fragment (8), sorti d'un éditorial du député UMP Jacques Myard, parle d'un "ghetto refermé sur lui-même ", c'est-à-dire volontairement fermé et isolé. Autrement dit, le ghetto y est vu comme un espace dans lequel les jeunes (« Français malgré eux ») se 
complaisent délibérément et dont ils refusent de sortir. Il en va de même dans l'extrait (9), repris à Jean-Pierre Moreau, l'ami d'un homme battu à mort par quelques jeunes à Stains". Celui-ci emploie le verbe devenir et le sujet impersonnel ça ("ça commençait à devenir un peu " ghetto $\left.»^{10} »\right)$ pour parler du processus de ghettoïsation. Comme devenir, d'après Le Petit Robert, c'est " passer d'un état à (un autre), commencer à être (ce qu'on n'était pas) ", ce verbe attributif nous semble impliquer un processus de ghettoïsation endogène.

Bref, les fragments (4)-(9) démontrent que, si ghettos il y a dans tous les cas, le rôle qu'occupent les jeunes balance entre celui de victimes et celui de coupables. C'est le cotexte, plutôt que le domaine source de la métaphore même, qui détermine la représentation véhiculée. La métaphore ghetto n'est à chaque fois que façonnée d'une telle façon qu'elle rentre dans le patron argumentatif du journaliste (ou de la voix en question). Une même métaphore formelle, évoquant le même domaine source, peut donc impliquer des nuances évaluatives différentes, en fonction du positionnement des acteurs sur les pôles de l'axe de causalité. C'est le cotexte qui nous donne des indices sur cette distribution spécifique.

Mentionnons encore que certains textes dans lesquels on parle des banlieues en termes de ghettos offrent une vision non catégorique mais plus nuancée de la situation: ils prêtent attention aussi bien aux causes exogènes qu'aux origines endogènes de la ghettoïsation.

(10) Etre "de gauche», c'est chercher à desserrer les étaux dans lesquels des gens sont tenus. Les voyous maintiennent l'ordre et ont besoin de confirmer l'enfermement dans les ghettos où se forge une sorte d'oppression spécifique par la force et les illégalismes. La gauche lutte pour la sécurité, non parce que la droite le lui dicte, mais parce que l'insécurité rend la solidarité plus difficile, le changement social moins désirable. (Libération, 08/11/2005_28; nous soulignons)

(11) Les hommes politiques, de tous bords jouent avec le feu, qui font un argument électoraliste d'un problème fondamental de la société française. La spirale de la ghettoïsation s'aggrave, sous l'effet du chômage de masse, et celui de replis identitaires sur le territoire, la religion et l'origine ethnique. Elle s'accompagne de dérives criminelles, et de la fuite hors la loi de jeunes à l'agressivité sans objet ni cible que la volonté d'exclure tout ce qui ne fait pas partie de "leur" monde. [...] La droite et la gauche se sont également cassé le nez sur la réalité des ghettos, souvent minimisée et souvent oubliée des priorités. Quel gouvernement a fait des cités une grande cause nationale ? Or chacun sait que les murs des ghettos ne pourront être abattus que par une action déterminée, sur la durée, à ras le bitume, loin des caméras de TV, et sur tous les fronts, économique, social, culturel (et, bien sûr, policier), impliquant les habitants des cités eux-mêmes. Il faut commencer par briser le cycle rébellion-répression, parce qu'il ne peut être un remède à ce mal des banlieues, et qu'il faut enrayer d'urgence le risque de contagion. (Libération, 02/11/2005_2 ; nous soulignons)

En effet, en (10), les banlieues sont vues comme des lieux d'enfermement (terme qui implique une cause externe de la ghettoïsation, un facteur qui enferme). Or, le journaliste admet tout aussi bien que les "voyous" se complaisent et veulent maintenir cette situation d'enfermement (cause interne). Une même vision nuancée se trouve dans l'extrait (11) : le journaliste avoue que les banlieues sont des ghettos (« la spirale de la ghettoïsation », "la réalité des ghettos») et ceci partiellement à cause de facteurs exogènes ("chômage de masse »), mais aussi internes ("replis identitaire sur le territoire, la religion et l'origine ethnique », « dérives criminelles », « la fuite hors la loi de jeunes à l'agressivité », « exclure tout ce qui ne fait pas partie de "leur" monde »). De la 
même façon, comme le démontre la seconde partie du fragment («la droite et la gauche se sont également cassé le nez sur la réalité des ghettos »), le journaliste se positionne de façon aussi critique vis-à-vis de la droite que vis-à-vis de la gauche. A notre avis, s'il parle des banlieues en termes de ghettos et de contagion, c'est parce que ceci rentre bien dans son argumentation qui appelle aussi bien la droite que la gauche à l'action déterminée : c'est à l'aide de tels termes dramatistes que l'on se rendra compte que l'action est urgente, que les murs des ghettos doivent être abattus.

En d'autres termes, nous espérons avoir illustré à l'aide de la discussion de la métaphore ghetto comment une même métaphore formelle peut non seulement servir deux buts représentatifs opposés (victimisante et culpabilisante), mais également s'intégrer dans une représentation plus intermédiaire, nuancée et équilibrée des banlieues.

\section{Conclusion}

En analysant les métaphores utilisées dans la couverture médiatique de la «crise des banlieues ", nous avons remarqué que l'opposition des représentations (culpabilisantes versus victimisantes) qui parcourt, dans une mesure variable, le débat sur les banlieues ne débouche pas sur une opposition bien délimitable au niveau des métaphores, c'est-à-dire sur une opposition entre domaines source typiquement de droite (culpabilisants) et domaines source typiquement de gauche (victimisants). Aussi l'idée d'« un duel au niveau des préférences métaphoriques » (Eubanks $2000: 8,11$ ) et des domaines source évoqués, s'avère-t-elle trop simpliste pour être appliquée aux processus subtils dont il est question dans notre corpus de presse écrite. Plus particulièrement, nous avons constaté (et essayé d'illustrer à l'aide des occurrences de la métaphore ghetto), que le trajet argumentatif plus large du locuteur, duquel le cotexte immédiat de la métaphore donne des indices et dans lequel l'axe de causalité (avec ses pôles de responsabilité et de réactivité) joue un rôle-clé, a une influence sur les métaphores et leur «fonction représentative ». Cette influence cotextuelle rend clair qu'une approche bifurquée de la métaphore, qui ne tient compte que de l'interaction entre le domaine source et le topic sans prendre le cotexte plus large en considération, est inévitablement déficiente. Une classification des métaphores qui se limite à leurs domaines source et domaines cible, comme c'est souvent le cas dans des analyses à la Lakoff et Johnson, risque, d'après nous, de manquer des différences subtiles, mais importantes au niveau des représentations et évaluations véhiculées.

31 En résumé, en examinant les métaphores dans un discours médiatique bien spécifique, cet article a illustré comment une analyse cotextuelle des métaphores est un élément indispensable à une meilleure compréhension de la nature complexe de la métaphore dans le langage de la presse écrite. Aussi suivons-nous la perspective de Stern (1999) qui pose que :

The aspects of a source domain that are relevant to a target domain are heavily dependent not only on the domains themselves, but on the whole context of the utterance, even of the discourse (Stern (1999) paraphrasé dans Croft et Cruse (2004: 210)

32 Ou comme le pose Eubanks (2000: 167) de façon aussi concise que correcte: "To study metaphor should always be to study something else" (« l'étude des métaphores ne peut qu'être l'étude de quelque chose d'autre »; nous traduisons). 


\section{BIBLIOGRAPHIE}

Aponte Moreno, Marco, 2009, Metaphors in Hugo Chávez’s Political Discourse, VDM Verlag.

Boyer, Henri et Lochard, Guy, 1998, Scènes de télévision en banlieue 1950-1954, Paris,

L'Harmattan.

Cameron, Lynne, 2003, Metaphor in educational discourse, London, Continuum.

Cameron, Lynne, 2007, "Patterns of metaphor use in reconciliation talk", Discourse \& Society, 18, 2, p. 197-222.

Carston, Robyn, 2002, "Metaphor, ad hoc concepts and word meaning -more questions than answers", UCL Working Papers in Linguistics, 14, 2002, p. 83-105.

Charon, Jean-Marie, 2005, La presse quotidienne, Paris, La Découverte.

Chilton, Paul, 2004, Analysing Political Discourse. Theory and Practice, London, Routledge.

Coupland, Nikolas, 1999, “ “Other’ Representation”, in Verschueren, J., Östman, J-O., Blommaert, J. et Bulcaen, C. (éds), Handbook of Pragmatics, Amsterdam, John Benjamins, p. 1-24.

Croft, William et Cruse, D. Alan, 2004, Cognitive Linguistics, Cambridge, Cambridge

University Press.

Dijk, Teun A. Van, 1988, News as Discourse, Hillsdale, Lawrence Erlbaum.

Djordjevic, Ksenija, 2007, “Violence urbaine : lorsque la presse en parle”, in Boyer, H. (éd.), Stéréotypage, Stéréotypes : fonctionnements ordinaires et mises en scène.Tome 1 :Média (tisation)s, Paris, L'Harmattan, p. 135-150.

Eubanks, Philip, 2000, A war of words in the discourse of trade. The rhetorical constitution of metaphor, Carbondale, Southern Illinois University Press.

Fowler, Roger, 1991, Language in the news. Discourse and Ideology in the Press, London, Routledge.

Garcin-Marrou, Isabelle, 2007, “Des « jeunes » et des « banlieues » dans la presse de l'automne 2005 : entre compréhension et relégation”, Espaces et sociétés, 128-129, p. 23-37.

Koller, Veronika, 2003, "Metaphor Clusters, Metaphor Chains: Analyzing the multifunctionality of Metaphor in Text", Metaphorik.de, 5, p. 115-134.

Koller, Veronika, 2004, Metaphor and Gender in Business Media Discourse: a Critical Cognitive Study, New York, Palgrave MacMillan.

Lakoff, George, 2004, Don't think of an elephant. Know your values and frame the Debate, White River Junction, Chelsea Green Publishing.

Lakoff, George et Johnson, Mark, 1980, Metaphors we live by, Chicago, University of Chicago Press.

Lakoff, George et Johnson, Mark, 1999, Philosophy in the flesh: the embodied mind and its challenge to western thought, New York, Basic Books.

Le Monde, 2002, Le style du Monde, Guide rédactionnel. 
Moirand, Sophie, 2006, "Le choc des discours dans la presse française : de la crise des banlieues à celle des universités (novembre 2005, mars 2006)", communication d'ouverture au Premier colloque international de la FATFA, Adelaide, Australie (juillet 2006), publiée dans les actes du colloque en Australie.

Musolff, Andreas, 2004, Metaphor and political discourse: analogical reasoning in debates about Europe, Basingstoke: Palgrave Macmillan.

Olza Moreno, Inés, 2008, "Metáfora y argumentación en el lenguaje político y la prensa españoles. Aproximación a las metáforas relacionadas con el « proceso de paz »”, ELUA, 22, p. 213-242.

Pan, Zhongdang et Kosicki, Gerald M, 1993, "Framing Analysis: An Approach to News Discourse", Political Communication, 10, p. 55-75.

Peeters, Stefanie, 2010 (à paraître), "Metaphors in media discourse: from a conceptual metaphor approach to the relevance-theoretic "continuity view" (and back again)", in Witczak-Plisiecka, Iwona (éd.), Pragmatic Perspectives on Language and Linguistics 2009. vol. 1: Speech Actions in Theory and Applied Studies, Cambridge, Cambridge Scholars Publishing, p. 324-355.

Peralva, Angelina et Macé, Eric, 2002, Médias et violences urbaines. Débats politiques et construction journalistique, Paris, La documentation française.

Richards, Ivor A., 1936, The Philosophy of Rhetoric, New York, Oxford University Press.

Santa Ana, Otto, 1999, “ "Like an animal I was treated": anti-immigrant metaphor in US public discourse", Discourse and Society, 10, 2, p. 191-224.

Sedel, Julie, 2009, Les medias et la banlieue, Paris, Editions Le Bord de l'Eau/INA.

Semino, Elena, 2008, Metaphor in Discourse, Cambridge: Cambridge University Press.

Sperber, Dan et Wilson, Deirdre, 2008, “A deflationary account of metaphors”, in Gibbs, Raymond W. (éd.), The Cambridge Handbook of Metaphor and Thought, Cambridge, Cambridge University Press.

Van Gorp, Baldwin, 2006, Framing asiel, Acco, Leuven.

Vázquez Bermúdez, Miguel Angel, 2006, “Los medios toman partido”, Ámbitos, 15, p. 257-267.

Vega Moreno, Rosa E., 2007, Creativity and Convention. The Pragmatics of Everyday Figurative Speech, Amsterdam, John Benjamins.

Verschueren, Jef, 1985, International News Reporting. Metapragmatic Metaphors and the U-2, Amsterdam, John Benjamins.

Wilson, Deirdre et Carston, Robyn, 2007, “A unitary approach to lexical pragmatics:

Relevance, inference and ad hoc concepts", http://www.phon.ucl.ac.uk/home/lexprag07/pubs/ Wilson-Carston-Advances-14September06.doc.

Wilson, Deirdre et Sperber, Dan, 2004, "Relevance Theory", in Horn, Laurence R. et Ward, Gregory L. (éds), The Handbook of Pragmatics, Malden MA, Blackwell, p. 607-632.

Wodak, Ruth et de Cillia, Rudolf, 2006, "Politics and Language: Overview", in Brown, Keith (éd.), Encyclopedia of Language and Linguistics, 9, Oxford, Elsevier, p.

707-719.

Références primaires 
Auteur non mentionné, « Ils ont dit... », L'Humanité, 03/11/2005.

Auteur non mentionné, « Mots de ghetto », Libération, 05/11/2005.

Auteur non mentionné, « Les faits du jour », Libération, 08/11/2005.

Auteur non mentionné, « Emeutes : Paris se protège de la contagion. », Le Figaro,

$12 / 11 / 2005$.

Defait, Vincent, Dugrand, Maud et Sellem, Jacqueline, « Ils témoignent et ils proposent ", L'Humanité, 04/11/2005.

Fache, Alexandre, «L'insécurité, c'est une coproduction », L'Humanité, 05/11/2005.

Langhe, Anne-Charlotte de, « Le premier mort des émeutes », Le Figaro,

$08 / 11 / 2005$

Myard, Jacques, « Assez d'angélisme, adoptons nos méthodes répressives sans

mollir ! », Le Figaro, 04/11/2005.

Peyrat, Didier, « Banlieues : Mai 68 ou Weimar ? », Libération, 08/11/2005.

Sabatier, Patrick, « Contagion », Libération, 02/11/2005.

Sandid, Mustapha, «L'invité de la semaine Mustapha Sandid », L’Humanité,

$07 / 11 / 2005$

\section{NOTES}

1. Notre corpus contient non seulement des textes journalistiques traditionnellement conçus comme purement informatifs, factuels et, du coup, objectifs (brèves, synthèses, reportages,...), mais aussi des textes qui se profilent explicitement comme argumentatifs et subjectifs (éditoriaux, colonnes d'opinion). Toutefois, nous devons ajouter, à ce sujet, que même le discours journalistique informatif ne peut se détacher d'une certaine subjectivité, d'une articulation (plus ou moins ouverte) de certaines intentions persuasives, valeurs ou idées idéologiques, tout comme l'ont démontré entre autres Verschueren (1985 : chap.1), Van Dijk (1988 : 119-125), Vázquez Bermúdez (2006) et Olza Moreno (2008 : 214).

2. Voir e.a. les études de Santa Ana 1999; Aponte Moreno 2009, pour n'en énumérer que quelques-unes.

3. Cf. Eubanks (2000: 16): «[m]etaphor theorists have done fine brushwork on an Aristotelian canvas. And the Aristotelian paradigm has been detailed with extraordinary care and creativity $[\ldots] "$

4. A quelques exceptions près : ainsi, dans la théorie de la pertinence, dans sa version la plus récente (Carston 2002; Wilson et Sperber 2004; Vega Moreno 2007; Wilson et Carston 2007; Sperber et Wilson 2008), la métaphore n'est plus considérée en termes d'un "mapping » entre deux domaines qui se base sur les principes d'analogie/similarité. Au contraire, les théoriciens de la pertinence préfèrent approcher la métaphore comme étant l'affaire d'un seul concept lequel est élargi pour y inclure des entités qui n'y appartiennent conventionnellement pas. De cette façon, la théorie de la pertinence réussit à proposer une explication unifiée tant pour les sens littéraux que pour les sens dits figurés, vision unifiée qu'on désigne conventionnellement par le terme vision de continuité (« continuity view »). Voir également Peeters (2010).

5. En effet, si Le Figaro s'avère un journal plutôt conservateur, Libération se situe plus à gauche du spectre idéologique, L'Humanité se positionnant même à l'extrême gauche. Le Monde, quant à lui, 
s'autoproclame dans son "contrat de lecture» (Le Style du Monde, 2002) «un quotidien indépendant qui n'obéit qu'à ses propres critères pour définir son traitement de l'information. [... ] un quotidien pluraliste ».

6. Evidemment, nous ne pouvons oublier que les journalistes ne sont pas les seuls énonciateurs présents dans les textes journalistiques, polyphoniques par nature. En effet, le comportement verbal de personnages publics et certains événements communicatifs pertinents constituent des objets-clés pour les journalistes (cf. Verschueren 1985). Par conséquent, toute analyse du discours médiatique doit prendre deux niveaux distincts mais hautement interdépendants en considération : le discours dit secondaire (des journalistes mêmes) et le discours dit primaire (des hommes politiques, témoins oculaires, porte-parole de toutes sortes, etc.), dont les journalistes parlent et lequel ils commentent. Comme les journalistes sont toujours simultanément des producteurs et des reproducteurs d'information, le texte journalistique se transforme en un « collage hybride de différentes composantes originales et recontextualisées » (Koller 2003 : 120).

7. De cette façon, notre corpus semble confirmer les analyses antérieures à 2005 sur la couverture médiatique des banlieues (comme Peralva et Macé (2002)), lesquelles discernent deux tendances opposées dans la représentation des banlieues et leurs problèmes : l'angélisme, d'un côté, et le réalisme, de l'autre. Voir, à ce sujet, également les observations de Garcin-Marrou (2007) et Sedel (2009: 130-133).

8. Cependant, l'on ne peut pas non plus penser que la distinction entre représentation victimisante - représentation culpabilisante des banlieues coïncide avec la distinction entre presse de gauche- presse de droite. En fait, la situation est assez nuancée. Ou comme le mentionne Sedel (2009: 132) : "La démarcation entre "positif» et "négatif », "réaliste», " angéliste", n'est pas réductible aux titres de presse dans la mesure où elle traverse les rédactions, les services et varie d'un journaliste à un autre ».

9. Vendredi le 4 novembre 2005, Jean-Jacques Le Chenadec, un homme de 61 ans, et son ami JeanPierre Moreau ont été agressés par quelques jeunes en bas de leur immeuble à Stains. Tombé dans le coma, Le Chenadec est décédé quelques jours plus tard. D'où le titre significatif de cet article du Figaro: «Le premier mort des émeutes", phrase qui suggère que d'autres morts peuvent suivre.

10. Il est question ici d'un discours oral, repris par la journaliste à autrui (en l'occurrence, à JeanPierre Moreau). Il est intéressant de voir que la journaliste tente de rendre orthographiquement, par l'intermédiaire de guillemets, que Jean-Pierre Moreau a d'une façon ou d'une autre mis la métaphore ghetto en relief dans son discours (soit par la prosodie, soit physiquement en faisant le signe des guillemets avec ses doigts).

\section{RÉSUMÉS}

Sur la base d'une analyse des métaphores employées dans la presse écrite française lors des «violences urbaines» de 2005, cet article posera qu'une approche des métaphores axée sur le cotexte doit contrebalancer une tendance courante qui, inspirée par l'approche de Lakoff et Johnson (1980), met trop l'accent sur le domaine source de la métaphore et sur la façon dont celui-ci représente ou "cadre» le topic sous discussion. L'analyse de ghetto, une métaphore récurrente dans notre corpus, prouvera que, quoiqu'étant un facteur important, le domaine source de la métaphore est loin de suffire à lui seul pour rendre compte de la représentation que peut véhiculer cette métaphore. 
Based on the analysis of the metaphors that were used in the French written press coverage of the 2005 "riots", this article will defend the following thesis: a co-text-focused metaphor approach should counterbalance the popular tendency in metaphor studies, which, inspired by Lakoff and Johnson (1980), pays major attention to the metaphor's source domain and to the way in which this represents or "frames" the topic under discussion. The analysis of ghetto, a recurrent metaphor in our corpus, will illustrate that, while being an important factor, the source domain of a metaphor does not suffice to account for the representation this metaphor might convey.

\section{INDEX}

Mots-clés : évaluation, métaphore, cadrage, presse quotidienne, représentation, violence urbaine

Keywords : daily press, metaphor, framing, suburban violence

\section{AUTEUR \\ STÉFANIE PEETERS}

Université d'Anvers, Belgique 